Knegt et al.

\title{
Host plant quality mediates competition between arbuscular mycorrhizal fungi
}

Bram Knegt ${ }^{1,2}$, Jan Jansa ${ }^{3}$, Oscar Franken ${ }^{2}$, Daniel J. P. Engelmoer ${ }^{2}$, Gijsbert D. A. Werner ${ }^{2}$, Heike Bücking ${ }^{4}$ and E. Toby Kiers ${ }^{2}$

${ }^{1}$ Institute for Biodiversity and Ecosystem Dynamics, University of Amsterdam, P.O. box 94248, 1090 GE Amsterdam, Netherlands; ${ }^{2}$ Department of Ecological Science, Vrije Universiteit, $1081 \mathrm{HV}$, Amsterdam, Netherlands; ${ }^{3}$ Institute of Microbiology, Academy of Sciences of the Czech Republic, Vídeňská 1083, Praha 4, 14220, Czech Republic; ${ }^{4}$ Biology and Microbiology Department, South Dakota State University, Brookings, SD 57007, USA

Corresponding author: Bram Knegt

Institute for Biodiversity and Ecosystem Dynamics, University of Amsterdam

P.O. box 94248

1090 GE Amsterdam, Netherlands

Email: a.j.knegt@uva.nl

Telephone: 0031-6-22753095

Received:

Revision received:

Accepted:

Corresponding editor:
05 June 2014

28 August 2014

16 September 2014

Thorunn Helgason 
Knegt et al.

\begin{abstract}
Arbuscular mycorrhizal fungi exchange soil nutrients for carbon from plant hosts. Empirical works suggests that hosts may selectively provide resources to different fungal species, ultimately affecting fungal competition. However, fungal competition may also be mediated by colonization strategies of the fungi themselves. To test whether host quality drives fungal colonization strategies, we allowed competing fungi access to the roots of plants that varied in quality (manipulated by shading). We used quantitative PCR and microscopy to assess fungal competitive dynamics and found that shaded plants were not left as an open niche for less competitive fungi. However, while competitive fungi outcompeted less competitive fungi, the intensity of this effect depended on the quality of the host, with the strongest differences found on low-quality (shaded) hosts. Our results suggest that environmental conditions for the host aboveground play a role in the competitive interactions among fungi belowground.
\end{abstract}

\title{
Key words
}

Biological market, cooperation, competitive exclusion, mutualism, priority effects, preferential allocation, qPCR, symbiosis

\section{Introduction}

Over $70 \%$ of all known plant species form partnerships with arbuscular mycorrhizal fungi (AMF), exchanging mineral nutrients for carbohydrates from plant hosts (Smith and Read 2008). From an ecological and evolutionary perspective, these exchange processes exhibit interesting dynamics. First, both plant and fungal partners can vary in mutualistic quality (Kiers and Denison 2008). The degree to which AMF benefit their hosts is highly contextdependent (Hoeksema et al. 2010), and in extreme cases AMF can even reduce host growth 
Knegt et al.

(Klironomos 2003; Jones and Smith 2004). Plant hosts can also vary in mutualistic quality, for example by providing less carbon to fungal partners when soil nutrient levels are high or when plant hosts are shaded and carbon limited (Treseder and Allen 2000, Grman 2012, Grman and Robinson 2013). Second, fungal competition can be intense (Herrera Medina et al. 2003, Kennedy 2010, Engelmoer et al. 2014). Initial colonization by fungi can prevent later colonization by others (Werner and Kiers 2014), even of the same species (Vierheilig 2004). Third, the mycorrhizal mutualism typically involves complex networks of simultaneous interactions among plant and fungal partners (Giovannetti et al. 2004, Mikkelsen et al. 2008). At any given time, plants are colonized by multiple AMF species (Jansa et al. 2008) and conversely, one AMF network can colonize multiple host plants and species (Selosse et al. 2006, Mikkelsen et al. 2008, Lekberg et al. 2010). Because of these many-to-many interactions, neither partner can be forced to cooperate; no single plant or AM fungus exclusively controls the nutrient supply of its symbiotic partner (Denison and Kiers 2011). Lastly, research has suggested that some AMF species that are beneficial at promoting host growth can be less competitive than AMF that are less beneficial, suggesting a fitness trade-off in plant growth promotion and competitive ability (Bennett and Bever 2009). Competition between AMF could thus lead to a decreased abundance of high-quality mutualistic partners for plants (Thonar et al. 2014).

Given these various constraints, researchers have asked how individual plants and fungi maintain high-quality interactions, i.e. with partners that provide most nutrients or otherwise enhance fitness (Bever et al. 2009, Lekberg et al. 2010, Denison and Kiers 2011, Hammer et al. 2011, Walder et al. 2012). One possibility is that partners can mediate competition in such a way that high-quality interactions are favored, leading to an increase in abundance of particular partners. It has been hypothesized that fungal competition is largely a host-driven process, with hosts providing different amounts of resources to fungal species, 
Knegt et al.

ultimately affecting the outcome of fungal competition (Pearson et al. 1993, Kennedy 2010, Werner and Kiers 2014). Recent work supporting this idea has shown that host plants can differentially allocate resources in their root systems, preferentially supporting the fungal species that provide the most nutrients (Bever et al. 2009, Kiers et al. 2011). In turn, fungal partners appear to increase nutrient transfer to high-quality plants, i.e. plants that provide more carbohydrates (Lekberg et al. 2010, Hammer et al. 2011, Kiers et al. 2011, Fellbaum et al. 2012), resulting in dynamics of trade between plants and fungi that resemble a biological market (Grman et al. 2012, Werner et al. 2014, Fellbaum et al. 2014). However, it is not well understood how these preferential rewarding mechanisms operate under natural conditions. Previous studies following nutrient exchange dynamics have largely relied on in vitro root organ cultures lacking a photosynthetic top (Bücking and Shachar-Hill 2005, Hammer et al. 2011, Kiers et al. 2011, Fellbaum et al. 2012). These have been criticized for underplaying the role of photosynthetic tissues, hormone regulation and source-sink relationships between plant organs (Fortin et al. 2002, Smith and Smith 2011). A second problem is that resource exchange studies generally use only one fungal species (but see Engelmoer et al. 2014), while in nature fungal communities rarely are monotypic (Kivlin et al. 2011). Given these limitations, it remains unknown how partner quality affects competitive dynamics in the mycorrhizal mutualism. Is host quality an important factor driving fungal colonization strategy? Will fungi compete to preferentially colonize a higher quality host or will a low quality plant host serve as a niche for less competitive fungi?

To answer these questions, we studied the competitive dynamics of closely related fungal species when fungi had access to high and low quality plant hosts. We manipulated plant quality through shading: when photosynthetic rate is reduced, plants are potentially less attractive to AMF for resource exchange (Heinemeyer et al. 2003, Fitter 2006, Kiers and Van der Heijden 2006), as it reduces net carbohydrate assimilation rates (Loach 1970). AMF 
Knegt et al.

colonizing the roots of shaded plants contain fewer structures for nutrient exchange (arbuscules), a potential indication of a reduced nutrient exchange with the host (Whitbeck 2001, Hodge and Fitter 2010).

We constructed experimental setups with two plants in a single microcosm, of which one plant was shaded. While the roots of host plants were separated with a mesh, hyphae were able to cross this barrier, allowing fungal networks to be formed between the two host plants. We inoculated plants with various combinations of two AMF species that differed in their colonization strategy (Table 1), and tested whether shading affected competitive interactions. Although colonizing a shaded host plant may still be beneficial for AMF, we expected the fungi to preferentially compete for a non-shaded host.

\section{Methods}

Plant, fungal and soil material

Medicago truncatula seedlings (variety Jemalong A17, courtesy of Dr. B. Hause, Leibniz Institute of Plant Biochemistry, Halle, Germany) were germinated following standard protocols (http://www.noble.org/medicagohandbook/), and transferred to sterilized peatbased soil to grow for $8 \mathrm{~d}$. Seedlings were assigned to three size classes (small, middle, large) and transplanted to sterilized polypropylene microcosms $(20 \times 11 \times 14 \mathrm{~cm}$, volume $3.0 \mathrm{~L}$, Fig S1), with size classes distributed equally among treatments. During transplantation, seedlings were inoculated with in vitro root cultures (Daucus carota) of two fungal species: Rhizophagus irregularis (Schenck and Smith; isolate 09, known previously as Glomus intraradices, Krüger et al. 2012; Mycovitro S.L. Biotechnología Ecológica, Granada, Spain) and Glomus aggregatum (Schenck and Smith; isolate 0165 from the Long Term Mycorrhizal Research Site, University of Guelph, Canada). R. irregularis is characterized by a high arbuscular colonization pattern, indicative of high nutrient transfer to the host, while Glomus 
Knegt et al.

aggregatum allocates more energy to storage, in the form of vesicles, and less to mineral uptake and transfer to its host (Kiers et al. 2011). To minimize the chance of inoculation failure, the inocula consisted of two parts: (i) $50 \mathrm{mg}$ roots from the Daucus carota in vitro root culture containing fungal spores and mycelium, and (ii) culture medium containing 8001000 spores, which we applied as liquid inoculum directly to host roots. Plants were grown in microcosms containing autoclaved low nutrient dune sand with a $\mathrm{pH}$ of $7.2,0.2 \%$ organic matter, $0.3 \mathrm{mg} \mathrm{kg}^{-1} \mathrm{P}\left(\mathrm{CaCl}_{2}\right.$-extracted $), 190 \mathrm{mg} \mathrm{kg}^{-1}$ total $\mathrm{N}$ and a gravimetric water holding capacity of $25 \%$ (Kiers et al. 2011). Each microcosm was divided by a $50 \mu \mathrm{m}$ nylon mesh (BioDesign Inc. of New York, Carmel, NY, USA) glued onto a PVC window in the middle of the microcosm. A layer of sterile low density polyethylene beads (Fardem Packaging, Edam, Netherlands) was placed on top of the sand to prevent cross-contamination and to reduce evaporation.

\section{Growth conditions}

The experiment was conducted in a growth chamber with a controlled humidity of $75 \%$, a day / night cycle of $16 \mathrm{hr} / 8 \mathrm{hr}$ and a temperature of $22^{\circ} \mathrm{C} / 17^{\circ} \mathrm{C}$. During daytime, nonshaded plants were grown under a light intensity of $313 \mu \mathrm{mol} \mathrm{m} \mathrm{m}^{-2} \mathrm{~s}^{-1}\left(\mathrm{SD}=32 \mu \mathrm{mol} \mathrm{m} \mathrm{m}^{-2} \mathrm{~s}^{-1}\right)$ at plant level. The photosynthetic capability of one plant from each microcosm was reduced by shading with a tent made of sterilized 70\% shade cloth (Nettenlijn Duranet, Cuijk, Netherlands), reducing the light intensity to an average of $120 \mu \mathrm{mol} \mathrm{m}^{-2} \mathrm{~s}^{-1}(\mathrm{SD}=32 \mu \mathrm{mol} \mathrm{m}$ ${ }^{2} \mathrm{~s}^{-1}$, average shade percentage of $\left.61 \%(\mathrm{SD}=12 \%)\right)$. The shade tent was open on two sides to prevent an accumulation of plant volatiles. Every 2 weeks plants received nutrients through a Hoagland solution containing $50 \%$ of the original $\mathrm{N}(105 \mathrm{ppm})$ and $25 \%$ of the original $\mathrm{P}$ (7.75 ppm) concentration (Hoagland and Arnon 1950), while soil moisture content was kept 
Knegt et al.

at $50 \%$. Microcosms were placed at random in the climate chamber, and randomized every two weeks.

\section{Treatments}

In the single species treatments, both the shaded and the non-shaded plant treatments were inoculated with the two fungal species separately to compare plant growth effects from the different fungi, as well as the response of each fungus to colonizing a shaded and non-shaded host (Table 1). In the mixed treatment, a mixed inoculum of the two fungal species was applied to both the shaded and the non-shaded plants. The mixed inoculum consisted of equal parts of both fungal species and had a total volume that was equal to the total volume of inocula in the other treatments. This allowed us to assess whether one fungus competitively excluded the other, and whether light conditions affected the outcome of this competition. In the crossover experiments, the shaded plant was inoculated with one AMF species and the non-shaded plant with the other, so each fungal species had to cross the mesh in order to reach the second plant. This allowed an assessment of whether the fungi would colonize the other root compartment and compete with an already established AMF, and whether light conditions would affect this competition.

\section{Harvest}

After $56 \mathrm{~d}$, plants were harvested over a $10 \mathrm{~d}$ period by separating shoot and root parts at soil level. Shoot length and shoot surface area were measured using an area meter (LI-COR, Model 3100, Lincoln, NE, USA). Dry weight was determined after drying plant shoots at 70 ${ }^{\circ} \mathrm{C}$ for a minimum of $48 \mathrm{hr}$. Roots were washed, weighed and cut into $\sim 0.5 \mathrm{~cm}$ pieces, and then divided into three samples. One sample was stored at $-20{ }^{\circ} \mathrm{C}$ and another in $10 \% \mathrm{KOH}$ for trypan blue staining. The remaining root sample was weighed, dried at $70{ }^{\circ} \mathrm{C}$ for a 
Knegt et al.

minimum of $48 \mathrm{hr}$ and weighed again. The dry/wet weight ratio of this root sample was used to calculate the total root biomass. AMF colonization of the roots was determined using two methods. Firstly, trypan blue staining and the magnified intersection method allowed to assess fungal colonization percentage and the presence of fungal structures (vesicles and arbuscules) at 100 random intersections (McGonigle et al. 1990). Secondly, colonization ratios between AMF species were determined using quantitative real-time PCR (qPCR). Fungal markers in the mitochondrial larger subunit region allowed for species-specific detection and quantification via a probe based reaction (Kiers et al. 2011, Engelmoer et al. 2014, Table S1). DNA was extracted from freeze-dried (48 hr) roots using a DNeasy Plant Mini Kit (Qiagen, Hilden, Germany). After homogenization, but before DNA isolation, a known amount of internal DNA standard (a plasmid containing a part of the African cassava mosaic virus genome) was added to each sample (Thonar et al. 2012). The recovery rate of this internal DNA standard was used to determine DNA extraction efficiency, showing an average of $16 \%(\mathrm{SD}=9 \%)$. Samples with a recovery rate $<1 \%$ were excluded from qPCR analysis. Removing these samples resulted in less than 10 replicates per treatment in some comparisons. Reactions were performed using the HOT FIREPol Probe qPCR Mix Plus (Solis BioDyne, Tartu, Estonia) system on an Opticon CFD-0200 DNA Engine (MJ Research, St. Bruno, Canada) following manufacturer recommendations. Briefly, an initial denaturation step of $95^{\circ} \mathrm{C}$ for 15 min was followed by 40 cycles at $95^{\circ} \mathrm{C}$ for $10 \mathrm{~s}$ and annealing and amplification step (see Table S1 for temperature) for $60 \mathrm{~s}$. Fluorescence patterns were analyzed using Opticon Monitor software v3.1.32 (Bio-Rad Laboratories, Hercules, CA, USA). To obtain quantification cycle $(\mathrm{Cq})$ values for all samples, fluorescence threshold values were set within the domain of exponential increase after subtraction of background fluorescence, which was determined as the average fluorescence between cycle three and eight. From the $\mathrm{Cq}$ values gene copy concentrations were calculated using standard 
Knegt et al.

curves (Fig S2). When the observed Cq value fell below the detection limit (Table S2), the detection limit was used as a stand-in value for that sample. Gene copy concentrations of all samples were corrected proportionally to DNA extraction efficiency, and subsequently expressed as target sequence copy number per mg dried root material. Because fungal tissue contains mitochondria, this quantification of mitochondrial target sequences provides speciesspecific information about the amount of fungal tissue (Engelmoer et al. 2014, Werner and Kiers 2014).

\section{Data analysis}

R v2.15.3 (2013) was used to perform statistical analyses. Plant length, shoot area and shoot biomass were analyzed using linear mixed models (LMMs) as implemented in package lme4 (Bates et al. 2013), while confirming that assumptions for independence, homogeneity and normality were met. Root biomass was analyzed using a generalized linear mixed model (GLMM) following a gamma distribution, implemented in package glmmADMB (Fournier et al. 2012, Skaug et al. 2012), because it was not normally distributed and showed heteroscedasticity between treatments. Microscopic measurements of fungal infection were expressed as proportions of colonized intersections containing vesicles or arbuscules. These proportions were analyzed using GLMMs following a binomial distribution, as implemented in lme4. Because the qPCR measurements of fungal abundance showed heteroscedasticity and were not normally distributed, these were analyzed using GLMMs following a gamma distribution. In addition, when analyzing the effects of shading on G. aggregatum abundance in crossover and control treatments, two separate models were employed, one in shaded and one in non-shaded conditions, because a full model including the interaction between light conditions and treatment still suffered from strong heteroscedasticity. Finally, in the crossover treatments, the number of occasions in which each fungus successfully colonized 
Knegt et al.

the second host plant was scored. These data were analyzed using a generalized linear model (GLM) following a binomial distribution.

Full models, containing all explanatory variables (light treatment, fungal treatment, seedling size and treatment time) and their two-way interactions were first specified. These models were subsequently simplified using backward simplification, omitting non-significant interaction and main effects one by one until only significant effects remained. Treatment time (56-65 d) was collinear with seedling size (small, middle, large) at the start of the experiment. Therefore, seedling size was removed as a covariate and discrimination between the effects of seedling size and treatment time is not possible. By design, measurements of plants and fungi growing in the same microcosm are correlated. Microcosm was therefore included as a random factor when comparing measurements from the same microcosm, and plant as a random factor nested in microcosm when comparing the abundance of two fungal species in the same root system. For LMMs and GLMs, the significance of interactions or main effects was assessed through likelihood ratio tests (LRTs) comparing nested models with and without this main effect or interaction. For GLMMs significance was assessed using Wald tests (Bolker et al. 2009), implemented in the lmtest package (Zeileis and Hothorn, 2002). For significant main effects with more than two levels post hoc tests were performed using package multcomp (Hothorn et al. 2008), while for significant interaction effects the package phia (de Rosario-Martinez 2013) was used.

Model validation was carried out by plotting residuals against fitted values for all models, allowing identification of outliers, negative fitted values or heterogeneity. In addition, the independence of a model was confirmed by plotting residuals against each covariate. For GLMMs following a binomial distribution, overdispersion was calculated as the sum of squared residuals divided by the residual degrees of freedom. When models were overdispersed, an observation level random intercept was included (Zuur et al. 2009). 
Knegt et al.

\section{Results}

Plant growth

Plant biomass was positively affected by AMF colonization, with both root (Wald F (2)= 17.37, $\mathrm{p}<0.001$ ) and shoot biomass (interaction of light and fungal treatment: $\chi^{2}(2)=6.96, \mathrm{p}$ $=0.031$ ) significantly increased by AMF inoculation (Fig 1). Plants that were colonized by AMF in the single species treatments grew $\sim 30 \%$ taller $\left(\chi^{2}(2)=19.52, \mathrm{p}<0.001\right)$ and had a $\sim 60 \%$ larger shoot area $\left(\chi^{2}(2)=29.22, \mathrm{p}<0.001\right)$ than non-mycorrhizal controls (Fig S3). Shaded plants grew $\sim 25 \%$ taller $\left(\chi^{2}(1)=21.82, \mathrm{p}<0.001\right)$ and had a $\sim 40 \%$ increased shoot area $\left(\chi^{2}(1)=31.13, \mathrm{p}<0.001\right)$ compared to non-shaded plants (Fig S3). No effect was found of shading on the shoot biomass of non-mycorrhizal plants or plants colonized by $G$. aggregatum. However, when plants were colonized by $R$. irregularis, there was a $\sim 25 \%$ higher shoot biomass of non-shaded plants compared with shaded plants (Fig 1A). There was no effect of shading on root biomass in any of these treatments (Wald $\mathrm{F}(1)=2.78, \mathrm{p}=0.101$, Fig 1B).

\section{Fungal colonization}

In the single species treatments, stereomicroscopical analysis showed that $R$. irregularis colonized $\sim 90 \%$ of the root system, whereas G. aggregatum only colonized $\sim 60 \%$ of the root system $($ Wald z $(1)=10.16, \mathrm{p}<0.001$, Fig $2 \mathrm{~A})$. In addition, G. aggregatum produced significantly more vesicles than $R$. irregularis (Wald z $(1)=8.81, \mathrm{p}<0.001$, Fig 2B), suggesting that G. aggregatum allocates higher amounts of resources to storage. There were no differences in arbuscule proportions (Wald $\mathrm{z}(1)=0.55, \mathrm{p}=0.584$ ), or fungal abundance measured by qPCR (Wald F $(1)=2.51, \mathrm{p}=0.123$ ) between the two fungal species. Shading had no effect on colonization percentage (Wald $\mathrm{z}(1)=-0.18, \mathrm{p}=0.854$ ), vesicle proportions 
Knegt et al.

(Wald $\mathrm{z}(1)=-0.42, \mathrm{p}=0.675)$, arbuscule proportions (Wald $\mathrm{z}(1)=0.20, \mathrm{p}=0.839)$ or on fungal abundance measured by $\mathrm{qPCR}($ Wald $\mathrm{F}(1)=0.43, \mathrm{p}=0.517)$ in single fungal species treatments.

\section{Fungal competition}

There was no difference in plant benefit conferred by the single and mixed fungal treatments: plant length $\left(\chi^{2}(2)=0.39, p=0.824\right)$, shoot area $\left(\chi^{2}(2)=1.11, p=0.575\right)$, shoot biomass $\left(\chi^{2}\right.$ $(2)=0.57, \mathrm{p}=0.753)$ and root biomass (Wald $\mathrm{F}(2)=0.66, \mathrm{p}=0.523)$ in the mixed treatment did not differ significantly from single fungal species treatments. When measuring fungal abundance using qPCR, there was no significant difference between total fungal abundance of the mixed treatment compared to total fungal abundance in the single species treatments (Wald F $(2)=1.76, \mathrm{p}=0.184$ ). Regardless of light conditions $R$. irregularis reached a higher abundance than G. aggregatum (Wald z $(1)=8.36, \mathrm{p}<0.001$, Fig 3A). However, the success of G. aggregatum was affected by light conditions: its abundance was consistently lower in shaded compared to non-shaded plants (Wald z $(1)=4.02, \mathrm{p}<0.001)$.

At harvest the abundance of both fungal species in plant roots on either side of the mesh was measured using $\mathrm{qPCR}$, and revealed that $R$. irregularis was more successful than G. aggregatum in colonizing the root system of the second host plant $\left(\chi^{2}(1)=25.36, p<\right.$ 0.001). The abundance of $R$. irregularis in the root system of the second plant was equivalent to its abundance in the first plant (Wald F $(1)=1.44, \mathrm{p}=0.240$ ) and also equivalent to its abundance in the single species treatment (Wald $\mathrm{F}(l)=1.52, \mathrm{p}=0.224$ ), suggesting it was not affected by competition with G. aggregatum. In contrast, G. aggregatum was detected colonizing the root system of the second plant in only one instance, and therefore the abundance of $G$. aggregatum in the root systems of first and second plants could not statistically compared. Light conditions did play a role in invasion succession: when a plant 
Knegt et al.

that was previously colonized by G. aggregatum was invaded by R. irregularis, G.

aggregatum experienced a significant decline in abundance in shaded plants $\left(\chi^{2}(1)=12.55, \mathrm{p}\right.$

$<0.001)$, but not in non-shaded plants $\left(\chi^{2}(1)=0.99, \mathrm{p}=0.319\right.$, Fig 3B). Regardless of light

conditions, $R$. irregularis reached higher abundance than G. aggregatum in the crossover

treatments $($ Wald $\mathrm{F}(1)=11.71, \mathrm{p}=0.001)$.

\section{Discussion}

Plants face variable environments above and below ground. This environmental variability affects the plants themselves, but also their symbiotic partners, such as AMF. Microbes have to cope and adapt to hosts that vary in the benefits they provide (Goh et al. 2013, Zheng et al. 2014). In this study we directly assessed how varying host quality affects competition between two arbuscular mycorrhizal fungi. We found that regardless of light conditions $R$. irregularis outcompeted G. aggregatum, because it reached higher abundance in both the mixed and crossover treatments. $R$. irregularis was able to reach a higher abundance than $G$. aggregatum even when it had to first cross the mesh barrier and then compete with already established G. aggregatum in the root. Contrary to our prediction, $R$. irregularis did not leave the shaded plant as an open niche for G. aggregatum, but rather aggressively colonized all available host roots (Fig 3A). This suggests that the availability of root space is important in driving the colonization strategy of $R$. irregularis, consistent with previous observations in plant-fungal interactions (Kennedy 2010, Engelmoer et al. 2014). One explanation for the consistent colonization of both high- and low-quality hosts by $R$. irregularis could be that AMF are obligate biotrophs, and colonizing low-quality hosts would provide the fungus with a continuous access to a carbon source when a high-quality host becomes unavailable, for example by pathogen or herbivore damage. 
Knegt et al.

Given that $R$. irregularis is a better competitor than $G$. aggregatum, does the plant mediate the competition between the fungi through differentially allocating more resources to one of them? We assessed this by growing plants in shaded environments, where carbon resources are scarce. If, when both fungi are present, plants would preferentially allocate resources to one of them, we would expect the change in fungal abundance between shaded and non-shaded conditions to be different for each fungus. If plants would not differentiate between the fungi and just spend less resources on mycorrhizal mutualisms, we would expect similar changes in fungal abundance between shaded and non-shaded conditions. We found that $G$. aggregatum colonization was reduced when in direct competition with $R$. irregularis, and the intensity of this effect depended on light conditions for the host (Fig 3AB). This suggests that the environmental conditions for the host aboveground can play an important role in the competitive interactions between fungi belowground. But why would shading have an effect on belowground fungal competition?

One possible explanation for why light conditions affect fungal competition is that shaded plants have a 'tighter' carbon budget than non-shaded plants and are more likely to control their root colonization (Schmitt et al. 2013a). Given a tighter carbon budget, we would predict plants to allocate more carbon to R. irregularis compared to G. aggregatum because previous tests in root organ cultures demonstrated that $R$. irregularis generally transfers more P per unit of C compared to G. aggregatum (Kiers et al. 2011). Additionally, we found that G. aggregatum produced more storage vesicles than $R$. irregularis (Fig 2B), which is generally indicative of a hoarding strategy rather than high nutrient transfer (Kiers et al. 2011). We reject the possibility that fungi respond to shading by providing more nutrients to stressed plants, because previous research has demonstrated that shading does not reduce the carbon costs per mycorrhizal nutrient benefit (Heinemeyer et al. 2003, Olsson et al. 2010). Rather, if plant carbon budgets are limited through shading, plants may have less 
Knegt et al.

carbon to allocate among competing fungal strains (Fitter 2006, Kiers and Van der Heijden 2006), and this could explain the reduced abundance of G. aggregatum that we observed when co-inoculated with $R$. irregularis under shade conditions. However, recent work by Zheng et al (2014) found that increased shading decreased preferential allocation by host plants (Allium vineale) to the best fungal partner, opening up questions of whether hosts allocation patterns are consistent across plant species with different life history traits (e.g. Verbruggen et al. 2012).

In our crossover treatments, fungi could first colonize the host plant where they were inoculated, and then, after crossing the mesh, they could compete with another (preestablished) fungus for access to the root system of a second plant. This crossover competition is different from our mixed treatment, and also different from typical competition studies where the inoculum contains multiple species and fungi compete directly from the start of the experiment. Under crossover conditions, G. aggregatum was very poor at colonizing root systems that were previously colonized by $R$. irregularis. This may be explained by priority effects (Wilson 1984, Pearson et al. 1993) - defined as when early arriving species have a competitive advantage over later arriving species (Alford and Wilbur 1985). Research has demonstrated that when two AMF species physically interact, their spread can effectively be blocked by one another (Hepper et al. 1988). This means that being the first AM fungus to colonize a root system can provide a competitive advantage (Vierheilig et al. 2000, Werner and Kiers 2014). While G. aggregatum was severely hindered by the previous colonization by $R$. irregularis, there was no evidence that previous $G$. aggregatum colonization posed any difficulties to a subsequent spread of $R$. irregularis. Therefore, priority effects are not the only factor in determining fungal success.

The differences between the two fungi in the crossover treatments could theoretically also be explained by (i) differences in inoculum potential or (ii) differences in ability of 
Knegt et al.

crossing the mesh. However, we reject the hypothesis that differences in inoculum potential could explain the observed differences in fungal abundance because we standardized the amount of inoculum applied to each plant. Previous research using these fungal species has demonstrated that adding more inoculum (spores and roots) to M. truncatula hosts did not result in an increased colonization intensity for either of these species (Kiers et al. 2011). We also reject the second alternative hypothesis because both fungi crossed the mesh at least once, demonstrating that the mesh did not present an absolute barrier to either fungus. Although fungi could potentially have different propensities to cross the mesh, such effects should then not depend on light conditions. Therefore, this cannot explain the differences in fungal abundance between shaded and non-shaded plants that we observed. Rather, our data suggest that differences in fungal abundance between shaded and non-shaded plants are likely due to competitive differences of the fungi. When plants are shaded and fungi compete, the net result is a decrease in G. aggregatum abundance. Because plants have to be strict in allocating $\mathrm{C}$, especially under shade conditions, allocation to $R$. irregularis is favored. Thus, in shaded plants these competitive dynamics are even more evident than compared to nonshaded conditions.

An important question for the interpretation of our results is whether our shading method introduced enough variation in plant quality to affect AMF. Previous work has found different effects of shading on AMF, including reduced root colonization (Tester et al. 1986, Hodge and Fitter 2010, Olsson et al. 2010), no effect (Johnson 1976, Facelli et al. 1999), and increased root colonization (Furlan and Fortin 1977). These reports suggest that plants can respond differently to shading, and do not consistently reduce their carbohydrate flux to belowground symbionts. However, Schmitt et al. (2013b) demonstrated that Medicago plants are sensitive to shading, and will increase shoot growth at the expense of root growth to compensate for the reduced photosynthesis. Recently researchers were also able to show that 
Knegt et al.

even when shading had no significant effect on plant biomass, shaded M. truncatula plants still received less nutrients from a common mycelial network compared to non-shaded plants (Fellbaum et al. 2014). In line with these observations, we found that shaded plants grew taller and increased their shoot surface area, but did not show significant biomass differences (Fig S3). Our work confirms previous host-quality manipulations (e.g. Heinemeyer et al. 2004, Fellbaum et al. 2014) showing that light-dependent plant responses can affect plant and fungal interactions. Future work should consider testing host plants across a shade gradient to determine the sensitivity of host plants and their fungal communities to shading.

Our work aimed to quantify colonization patterns of closely related fungal species. A current debate in the literature is how to most accurately measure fungal abundances (Gamper et al. 2008, Tellenbach et al. 2010, Shi et al. 2012, Gorzelak et al. 2012). Visual counts of colonization rates using staining/microscopic methods and qPCR measurements, which measure fungal gene copy numbers, can disagree (Duhamel et al. 2013). In the current study we found significant differences in root colonization percentage measured by visual counts between the two fungal species in the single species treatments (Fig 2A), but this difference was not significant as measured by qPCR. Researchers have sought to explain such discrepancies, and suggested that spatial heterogeneity of fungal tissues can cause a poor correlation between microscopy and qPCR measurements of fungal colonization (Hart et al. 2013). However, using the same fungal strains, positive relationships have been found between fungal gene copy number measured by qPCR and the dry weight of extraradical hyphae (Engelmoer et al. 2014, Werner and Kiers 2014). Hyphal dry weight, however, can be very laborious to measure. Therefore, we advocate for a two method complementary approach: microscopic measurements provide valuable information about root colonization percentage and fungal structures, while qPCR can be used best for abundance, especially under mixed fungal conditions. 
Knegt et al.

\section{Conclusion}

We demonstrated that host plant quality, experimentally manipulated through shading, can mediate competition between different AMF species in a whole plant model system. Given that much of the previous work manipulating host quality and studying allocation patterns has been performed using in vitro root organ cultures (Bücking and Shachar-Hill 2005, Hammer et al. 2011, Kiers et al. 2011, Fellbaum et al. 2012 ), this is an important test under more natural conditions. Our research stresses the role of the host in influencing the composition of fungal communities in the rhizosphere, and of the local host environment in structuring AMF communities in the soil. Using microbial systems to test ecological and evolutionary theory is gaining popularity (Werner et al. 2014). Despite its hidden nature, the mycorrhizal mutualism can potentially serve as a model system for understanding complex competitive interactions: competition and nutrient exchange occurring on a small spatial scale can be closely tracked, and quality of the partners and growth conditions can be easily manipulated. This allows a quantification of resource exchange under different ecological conditions, thereby elucidating the plasticity of competitive strategies.

\section{Acknowledgements}

This research was supported by grants from the Dutch Science Foundation (NWO: Meervoud 836.10.001 and Vidi 864.10.005 grants) and the European Research Council (ERC starting grant) to ETK and from the National Science Foundation (IOS award 1051397) to HB. JJ was supported by the Fellowship of J. E. Purkyne, Ministry of Education, Youth and Sports (LK11224), and the long-term development program RVO 61388971. 
Knegt et al.

\section{Literature Cited}

Alford, R. A., and H. M. Wilbur. 1985. Priority effects in experimental pond communities: competition between Bufo and Rana. Ecology 66:1097-1105.

Bates, D., Maechler, M., and B. Bolker. 2013. Lme4: linear mixed-effects models using S4 classes. R package version 0.999999-2. http://CRAN.R-project.org/package=lme4

Bennett, A. E., and J. D. Bever. 2009. Trade-offs between arbuscular mycorrhizal fungal competitive ability and host growth promotion in Plantago lanceolata. Oecologia 160:807-816.

Bever, J. D., Richardson, S. C., Lawrence, B. M., Holmes, J., and M. Watson. 2009. Preferential allocation to beneficial symbiont with spatial structure maintains mycorrhizal mutualism. Ecology Letters 12:13-21.

Bolker, B. M., Brooks, M. E., Clark, C. J., Geange, S. W., Poulsen, J. R., Stevens, M. H. H., and J-S. S. White. 2009. Generalized linear mixed models: a practical guide for ecology and evolution. Trends in Ecology and Evolution 24:127-135.

Bücking, H., and Y. Shachar-Hill. 2005. Phosphate uptake, transport and transfer by the arbuscular mycorrhizal fungus Glomus intraradices is stimulated by increased carbohydrate availability. New Phytologist 165:899-912.

Denison, R. F., and E. T. Kiers. 2011. Life histories of symbiotic rhizobia and mycorrhizal fungi. Current Biology 21:R775-R785.

Duhamel, M., Pel, R., Ooms, A., Bücking, H., Jansa, J., Ellers, J., Van Straalen, N. M., Wouda, T., Vandenkoornhuyse, P., and E. T. Kiers. 2013. Do fungivores trigger the transfer of defensive metabolites from host plants to arbuscular mycorrhizal hyphae? Ecology 94:2019-2029. 
Knegt et al.

Engelmoer, D. J. P., Behm, J. E., and E. T. Kiers. 2014. Intense competition between arbuscular mycorrhizal mutualists in an in vitro root microbiome negatively affects total fungal abundance. Molecular Ecology 23:1584-1593.

Facelli, E., Facelli, J. M., Smith, S. E., and M. J. McLaughlin. 1999. Interactive effects of arbuscular mycorrhizal symbiosis, intraspecific competition and resource availability on Trifolium subterraneum cv. Mt. Barker. New Phytologist 141:535-547.

Fellbaum, C. R., Gachomo, E. W., Beesetty, Y., Choudhari, S., Strahan, G. D., Pfeffer, P. E., Kiers, E. T., and H. Bücking. 2012. Carbon availability triggers fungal nitrogen uptake and transport in arbuscular mycorrhizal symbiosis. Proceedings of the National Academy of Sciences USA 109:2666-2671.

Fellbaum, C. R., Mensah, J. A., Cloos, A. J., Strahan, G. E., Pfeffer, P. E., Kiers, E. T., and H. Bücking. 2014. Fungal nutrient allocation in common mycorrhizal networks is regulated by the carbon source strength of individual host plants. New Phytologist 203:646-656.

Fitter, A. H. 2006. What is the link between carbon and phosphorus fluxes in arbuscular mycorrhizas? A null hypothesis for symbiotic function. New Phytologist 172:3-6.

Fortin, J. A., Bécard, G., Declerck, S., Dalpé, Y., St-Arnaud, M., Coughlan, A. P., and Y. Piché. 2002. Arbuscular mycorrhiza on root-organ cultures. Canadian Journal of Botany 80:1-20.

Fournier, D. A., Skaug, H. J., Ancheta, J., Ianelli, J., Magnusson, A., Maunder, M., Nielsen, A., and J. Sibert. 2012. AD Model Builder: using automatic differentiation for statistical inference of highly parameterized complex nonlinear models. Optimization Methods and Software 27:233-249. 
Knegt et al.

Furlan, V., and J. A. Fortin. 1977. Effects of light intensity on the formation of vesiculararbuscular endomycorrhizas on Allium cepa by Gigaspora calospora. New Phytologist $79: 335-340$.

Gamper, H. A., Young, J. P. W., Jones, D. L., and A. Hodge. 2008. Real-time PCR and microscopy: are the two methods measuring the same unit of arbuscular mycorrhizal fungal abundance? Fungal Genetics and Biology 45:581-596.

Giovannetti, M., Sbrana, C., Avio, L., and P. Strani. 2004. Patterns of below-ground plant interconnections established by means of arbuscular mycorrhizal networks. New Phytologist 164:175-181.

Goh, C., Veliz Vallejos, D. F., Nicotra, A. B., and U. Mathesius. 2013. The impact of beneficial plant-associated microbes on plant phenotypic plasticity. Journal of Chemical Ecology 39:826-839.

Gorzelak, M. A., Holland, T. C., Xing, X., and M. M. Hart. 2012. Molecular approaches for AM fungal community ecology: a primer. Journal of Microbiological Methods 90:108114.

Grman, E. 2012. Plant species differ in their ability to reduce allocation to non-beneficial arbuscular mycorrhizal fungi. Ecology 93:711-718.

Grman, E., and T. M. P. Robinson. 2013. Resource availability and imbalance affect plantmycorrhizal interactions: a field test of three hypotheses. Ecology 94:62-71.

Grman, E., Robinson, T. M. P., and C. A. Klausmeier. 2012. Ecological specialization and trade affect the outcome of negotiations in mutualism. The American Naturalist 179:567581.

Hammer, E. C., Pallon, J., Wallander, H., and P. A. Olsson. 2011. Tit for tat? A mycorrhizal fungus accumulates phosphorus under low plant carbon availability. FEMS Microbiology Ecology 76:236-244. 
Knegt et al.

Hart, M. M., Forsythe, J., Oshowski, B., Bücking, H., Jansa, J., and E. T. Kiers. 2013. Hiding in a crowd - does diversity facilitate persistence of a low-quality fungal partner in the mycorrhizal symbiosis? Symbiosis 59:47-56.

Heinemeyer, A., Ridgway, K. P., Edwards, E. J., Benham, D. G., Young, J. P. W., and A. H. Fitter. 2004. Impact of soil warming and shading on colonization and community structure of arbuscular mycorrhizal fungi in roots of a native grassland community. Global Change Biology 10:52-64.

Hepper, C. M., Azcon-Aguilar, C., Rosendahl, S., and R. Sen. 1988. Competition between three species of Glomus used as spatially separated introduced and indigenous mycorrhizal inocula for leek (Allium porrum, L.). New Phytologist 110:207-215.

Herrera Medina, M. J., Gagnon, H., Piché, Y., Ocampo J. A., García Garrido, J. M., and H. Vierheilig. 2003. Root colonization by arbuscular mycorrhizal fungi is affected by the salicylic acid content of the plant. Plant Science 164:993-998.

Hoagland, D. R., and D. I. Arnon. 1950. The water-culture method for growing plants without soil. California Agricultural Experiment Station Circular 347:1-32.

Hodge, A., and A. H. Fitter. 2010. Substantial nitrogen acquisition by arbuscular mycorrhizal fungi from organic material has implications for $\mathrm{N}$ cycling. Proceedings of the National Academy of Sciences USA 107:13754-13759.

Hoeksema, J. D., Chaudhary, V. B., Gehring, C. A., Collins Johnson, N., Karst, J., Koide, R. T., Pringle, A., Zabinsky, C., Bever, J. D., Moore, J. C., Wilson, G. W. T., Klironomos, J. N., and J. Umbanhowar. 2010. A meta-analysis of context-dependency in plant response to inoculation with mycorrhizal fungi. Ecology Letters 13:394-407.

Hothorn, T., Bretz, F., and P. Westfall. 2008. Simultaneous inference in general parametric models. Biometrical Journal 50:346-363. 
Knegt et al.

Jansa, J., Smith, F. A., and S. E. Smith. 2008. Are there benefits of simultaneous root colonization by different arbuscular mycorrhizal fungi? New Phytologist 177:779-789.

Jones, M. D., and S. E. Smith. 2004. Exploring functional definitions of mycorrhizas: are mycorrhizas always mutualisms? Canadian Journal of Botany 82:1089-1109.

Kennedy, P. G. 2010. Ectomycorrhizal fungi and interspecific competition: species interactions, community structure, coexistence mechanisms and future research directions. New Phytologist 187:895-910.

Kiers, E. T., and M. G. A. Van der Heijden. 2006. Mutualistic stability in the arbuscular mycorrhizal symbiosis: exploring hypotheses of evolutionary cooperation. Ecology $87: 1627-1636$.

Kiers, E. T., and R. F. Denison. 2008. Sanctions, cooperation and the stability of plantrhizosphere mutualisms. Annual Review of Ecology, Evolution and Systematics 39:215236.

Kiers, E. T., Duhamel, M., Beesetty, Y., Mensah, J. M., Franken, O., Verbruggen, E., Fellbaum, C. R., Kowalchuk, G. A., Hart, M. M., Bago, A., Palmer, T. M., West, S. A., Vandenkoornhuyse, P., Jansa, J., and H. Bücking. 2011. Mycorrhizal rewards stabilize cooperation in the mycorrhizal symbiosis. Science 333:880-882.

Kivlin, S. N., Hawkes, C. V., and K. K. Treseder. 2011. Global diversity and distribution of arbuscular mycorrhizal fungi. Soil Biology \& Biochemistry 43:2294-2303.

Klironomos, J. N. 2003. Variation in plant response to native and exotic arbuscular mycorrhizal fungi. Ecology 84:2292-2301.

Krüger, M., Krüger, C., Walker, C., Stockinger, H., and A. Schüßler. 2012. Phylogenetic reference data for systematics and phylotaxonomy of arbuscular mycorrhizal fungi from phylum to species level. New Phytologist 193:970-984. 
Knegt et al.

Lekberg, Y., Hammer, E. C. and P. A. Olsson. 2010. Plants as resource islands and storage units - adopting the mycocentric view of arbuscular mycorrhizal networks. FEMS Microbiology Ecology 74:336-345.

Loach, K. 1970. Shade tolerance in tree seedlings. New Phytologist 69:273-286.

McGonigle, T. P., Miller, M. H., Evans, D. G., Fairchild, G. L., and J. A. Swan. 1990. A new method which gives an objective measure of colonization of roots by vesicular arbuscular mycorrhizal fungi. New Phytologist 115:495-501.

Mikkelsen, B. L., Rosendahl, S., and I. Jakobsen. 2008. Underground resource allocation between individual networks of mycorrhizal fungi. New Phytologist 180:890-899.

Olsson, P. A., Rahm, J., and N. Aliasgharzad. 2010. Carbon dynamics in mycorrhizal symbioses is linked to carbon costs and phosphorus benefits. FEMS Microbial Ecology $72: 123-131$.

Pearson, J. N., Abbott, L. K., and D. A. Jasper. 1993. Mediation of competition between colonizing VA mycorrhizal fungi by the host plant. New Phytologist 123:93-98.

R Development Core Team. 2013. R: A language and environment for statistical computing. R Foundation for Statistical Computing, Vienna, Austria. ISBN 3-900051-07-0, http://www.R-project.org/.

de Rosario-Martinez, H. 2013. Phia: Post-hoc interaction analysis. Package version 0.1-3. http://CRAN.R-project.org/package=phia.

Schmitt, A., Pausch, J., and Y. Kuzyakov. 2013a. C and N allocation in soil under ryegrass and alfalfa estimated by ${ }^{13} \mathrm{C}$ and ${ }^{15} \mathrm{~N}$ labelling. Plant Soil 368:581-590.

Schmitt, A., Pausch, J., and Y. Kuzyakov. 2013b. Effect of clipping and shading on C allocation and fluxes in soil under ryegrass and alfalfa estimated by ${ }^{14} \mathrm{C}$ labelling. Applied Soil Ecology 64:228-236. 
Knegt et al.

Selosse, M., Richard, F., He, X., and S. W. Simard. 2006. Mycorrhizal networks: des liaisons dangereuses? Trends in Ecology and Evolution 21:621-628.

Shi, P., Abbott, L. K., Banning, N. C., and B. Zhao. 2012. Comparison of morphological and molecular genetic quantification of relative abundance of arbuscular mycorrhizal fungi within roots. Mycorrhiza 22:501-513.

Skaug, H., Fournier, D., Nielsen, A., Magnusson, A., and B. Bolker. 2012. Generalized linear mixed models using AD Model Builder. R package version 0.7.4.

Smith, S. E., and D. J. Read. 2008. Mycorrhizal symbiosis (3 ${ }^{\text {rd }}$ edition). Academic Press, Elsevier, Amsterdam.

Smith, S. E., and F. A. Smith. 2011. Roles of arbuscular mycorrhizas in plant nutrition and growth: new paradigms from cellular to ecosystem scales. Annual Review of Plant Biology 62:227-250.

Tellenbach, C., Grünig, C. R., and T. N. Sieber. 2010. Suitability of quantitative real-time PCR to estimate the biomass of fungal root endophytes. Applied and Environmental Microbiology 76:5764-5772.

Tester, M., Smith, S. E., Smith, F. A., and N. A. Walker. 1986. Effects of photon irradiance on the growth of shoots and roots, on the rate of initiation of mycorrhizal infection and on the growth of infection units in Trifolium subterraneum L. New Phytologist 103:375-390.

Thonar, C., Erb, A., and J. Jansa. 2012. Real-time PCR to quantify composition of arbuscular mycorrhizal communities - marker design, verification, calibration and field validation. Molecular Ecology Resources 12:219-232.

Thonar, C., Frossard, E., Šmilauer, P., and J. Jansa. 2014. Competition and facilitation in synthetic communities of arbuscular mycorrhizal fungi. Molecular Ecology 23:733-746.

Treseder, K. K., and M. F. Allen. 2000. Mycorrhizal fungi have a potential role in soil carbon storage under elevated $\mathrm{CO}_{2}$ and nitrogen deposition. New Phytologist 147:189-200. 
Knegt et al.

Verbruggen, E., El Mouden, C., Jansa, J., Akkermans, G., Bücking, H., West, S. A., and E. T. Kiers. 2012. Spatial structure and interspecific cooperation: theory and an empirical test using the mycorrhizal mutualism. The American Naturalist 179:E133-146.

Vierheilig, H., Garcia-Garrido, J. M., Wyss, U., and Y. Piché. 2000. Systemic suppression of mycorrhizal colonization of barley roots already colonized by AM fungi. Soil Biology and Biochemistry 32:589-595.

Vierheilig, H. 2004. Further root colonization by arbuscular mycorrhizal fungi in already mycorrhizal plants is suppressed after a critical level of root colonization. Journal of Plant Physiology 161:339-341.

Walder, F., Niemann, H., Natarajan, M., Lehmann, M. F., Boller, T., and A. Wiemken. 2012. Mycorrhizal networks: common goods of plants shared under unequal terms of trade. Plant Physiology 159:789-797.

Werner, G. D. A. and E. T. Kiers. 2014. Order of arrival structures arbuscular mycorrhizal colonisation of plants. New Phytologist, in press.

Werner, G. D. A., Strassmann, J. E., Ivens, A. B. F., Engelmoer, D. J. P., Verbruggen, E., Queller, D. C., Noë, R., Collins Johnson, N., Hammerstein, P., and E. T. Kiers. 2014. Evolution of microbial markets. Proceedings of the National Academy of Sciences USA $111: 1237-1244$.

Whitbeck, J. L. 2001. Effects of light environment on vesicular-arbuscular mycorrhiza development in Inga leiocalycina, a tropical wet forest tree. Biotropica 33:303-311.

Wilson, J. M. 1984. Competition for infection between vesicular-arbuscular mycorrhizal fungi. New Phytologist 97:427-435.

Zeileis, A., and T. Hothorn. 2002. Diagnostic checking in regression relationships. R News 2:7-10. 
Knegt et al.

Zheng, C., Ji, B., Zhang, J., Zhang, F., and J. D. Bever. 2014. Shading decreases plant carbon preferential allocation towards the most beneficial mycorrhizal mutualist. New Phytologist. doi: 10.1111/nph.13025.

Zuur, A. F., Ieno, E. N., Walker, N. J., Saveliev, A. A., and G. M. Smith. 2009. Mixed effects models and extensions in ecology with R. Statistics for biology and health. Springer, New York. 
Knegt et al.

Figure 1. Effects of shading and AMF on shoot (A) and root (B) biomass in non-mycorrhizal control plants and the single species treatments. Error bars show the standard error of means, letters indicate significant differences between treatments $(p<0.05)$. "n.s." indicates not significant, “**” indicates $\mathrm{p}<0.01$.

Figure 2. AMF colonization strategies of $R$. irregularis and G. aggregatum in the single species treatments. (A) Proportion of infected roots and (B) proportion of vesicles. Vesicle percentage is the percentage of infected intersections that contained vesicles. Data from shaded and non-shaded plants are pooled, because light conditions had no significant effect on infection percentage or vesicle percentage. Error bars show the standard error of means, “**” indicates $\mathrm{p}<0.01$.

Figure 3. Effects of light conditions on competition between fungi measured by qPCR. (A) Abundance of AMF in the mixed treatment. (B) G. aggregatum abundance in its single species treatment ("control"), or when challenged by R. irregularis in crossover experiments (“crossover"). Y-axes are logarithmic. Error bars show the standard error of means, letters indicate significant differences between treatments $(\mathrm{p}<0.05)$. 
Knegt et al.

Table 1. Experimental treatments. Single species treatments contained only one AMF species, plants in the mixed treatments were inoculated with a mix of both AMF species, while in the crossover treatments the shaded plant was inoculated with one AMF species and the nonshaded plant with the other. Each treatment contained 10 replicates.

\begin{tabular}{|ccc|}
\hline Treatment & Non-shaded & Shaded \\
\hline Non-mycorrhizal & None & None \\
Single species & R. irregularis & R. irregularis \\
Single species & G. aggregatum & G. aggregatum \\
Mixed & Mix & Mix \\
Crossover & R. irregularis & G. aggregatum \\
Crossover & G. aggregatum & R. irregularis \\
\hline
\end{tabular}


Knegt et al.

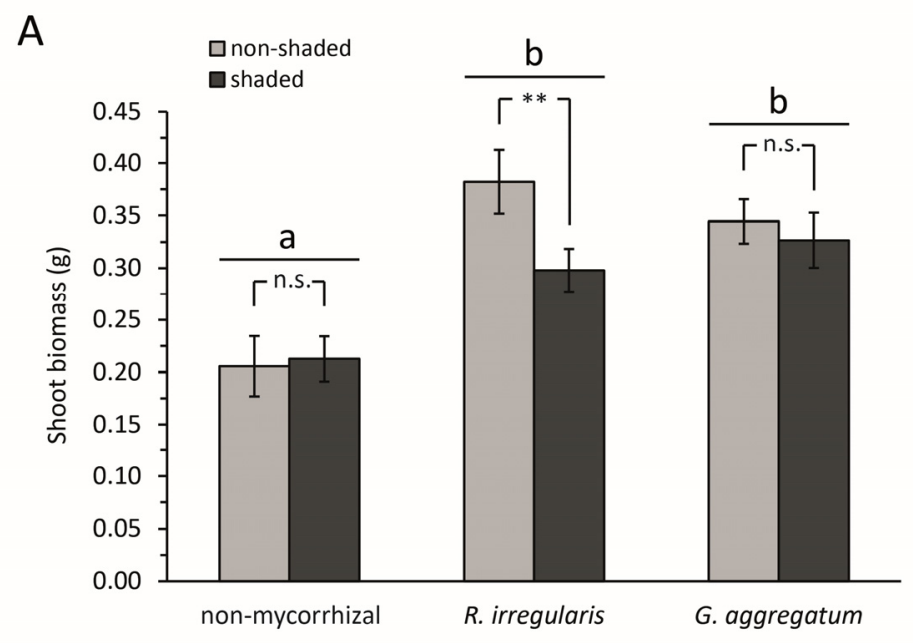

B

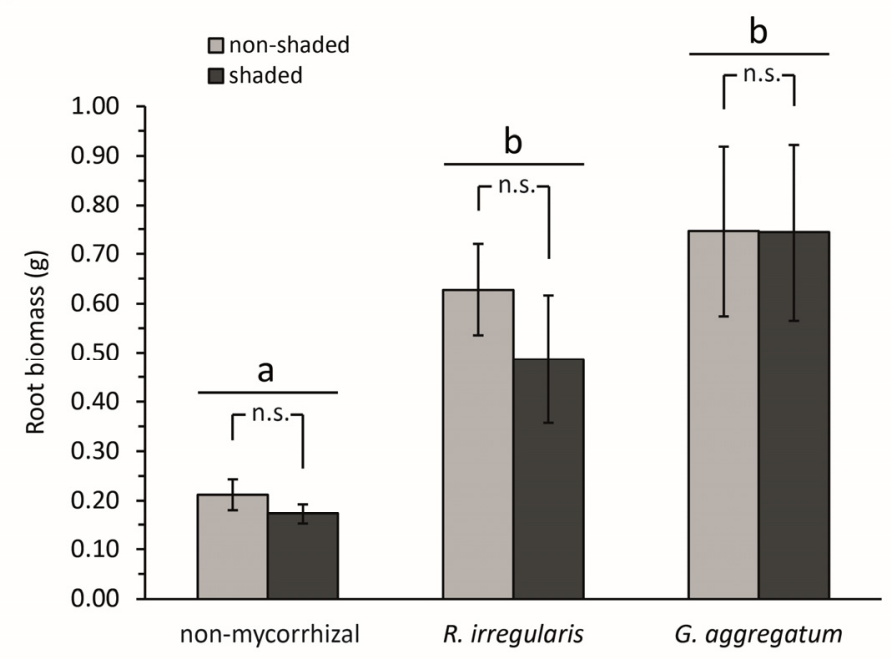

Figure 1. 
Knegt et al.
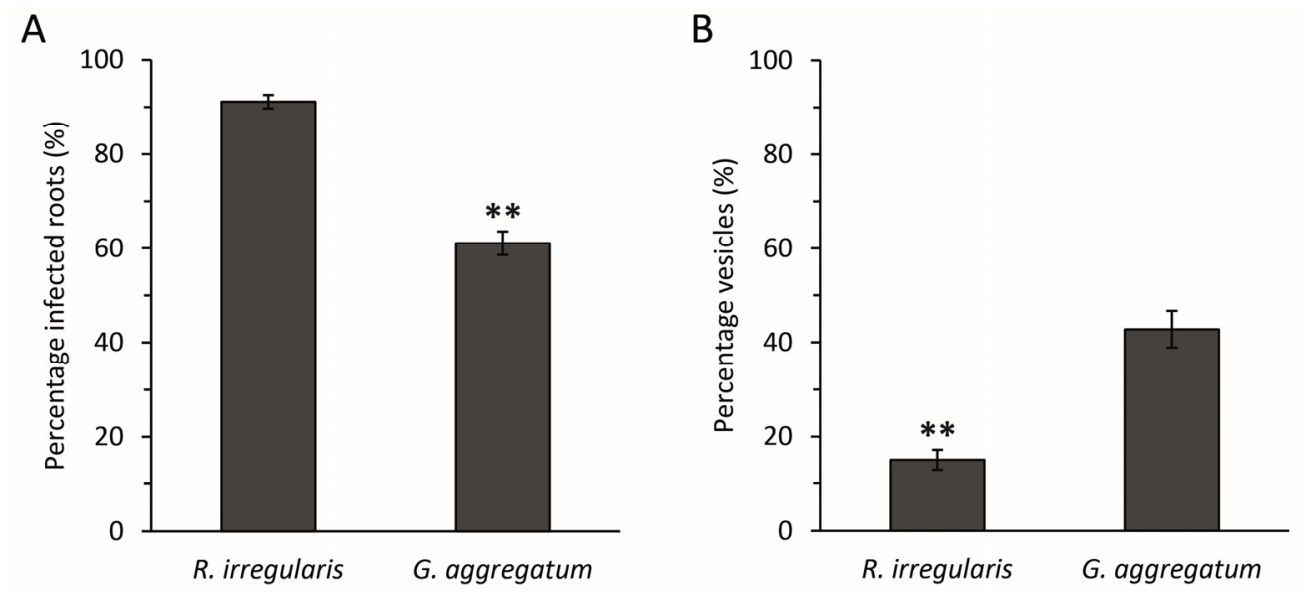

Figure 2. 
Knegt et al.
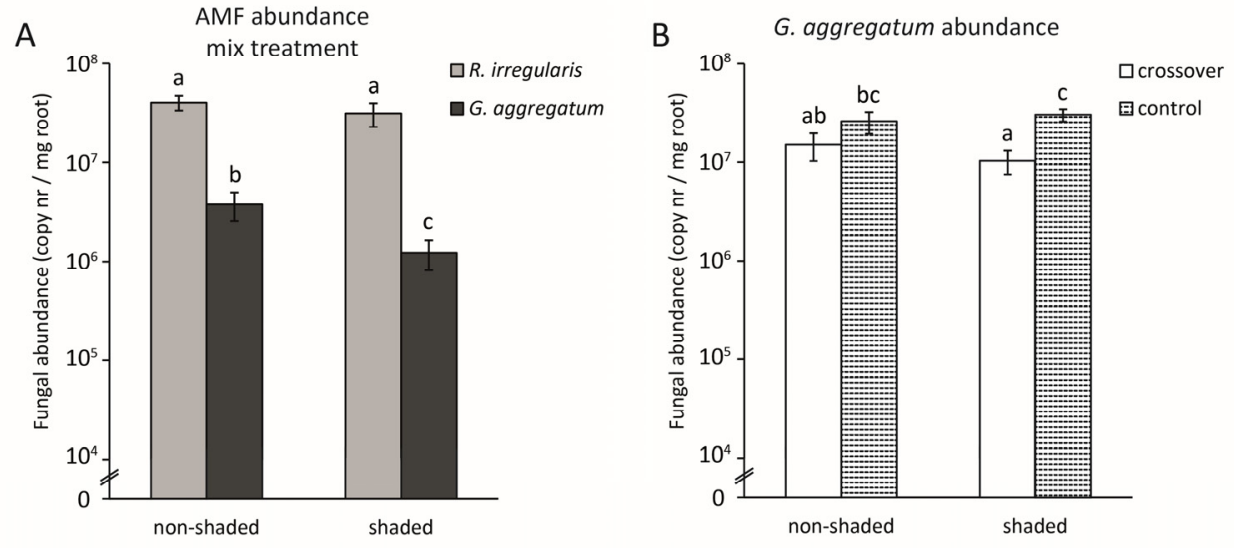

Figure 3. 